\title{
The Tailoring Of National Guidelines For Upper Extremity Post- Stroke Rehabilitation: A Concept In Use.
}

\author{
Salem F. Alatawi \\ University of Tabuk, sfalatawi@ut.edu.sa
}

Follow this and additional works at: https://nsuworks.nova.edu/ijahsp

Part of the Physical Therapy Commons, and the Physiotherapy Commons

This Manuscript has supplementary content. View the full record on NSUWorks here: https://nsuworks.nova.edu/ijahsp/vol17/iss1/11

\section{Recommended Citation}

Alatawi SF. The Tailoring Of National Guidelines For Upper Extremity Post-Stroke Rehabilitation: A Concept In Use.. The Internet Journal of Allied Health Sciences and Practice. 2019 Jan 01;17(1), Article 11.

This Manuscript is brought to you for free and open access by the College of Health Care Sciences at NSUWorks. It has been accepted for inclusion in Internet Journal of Allied Health Sciences and Practice by an authorized editor of NSUWorks. For more information, please contact nsuworks@nova.edu. 


\title{
The Tailoring Of National Guidelines For Upper Extremity Post-Stroke Rehabilitation: A Concept In Use.
}

\begin{abstract}
Background: In Saudi Arabia, national evidence-based clinical guidelines (NCG) were created based for healthcare interventions. However, clinicians appear to be slow to incorporate the evidence into practice. As a result, there exists a time-gap between the gathering and presentation of evidence for best practice and the use of that evidence by clinicians. It becomes, therefore, imperative to investigate the gap between the research evidence and clinical practice. The knowledge to action (KTA) framework is available to facilitate the implementation of research evidence. This framework incorporates the theory of tailoring, a concept that is intended to create a fit between the evidence for the intervention and its use by the clinician. Unfortunately, the process of tailoring stills awaits clear guidelines on how it should be implemented. Consequently, this study presents hitherto unknown strategies for using novel methods, which reflect the particular role of the KTA framework in tailoring recommendations. Aim: The purpose of this study is to examine the use of the UK National Clinical Guidelines for Stroke as a clinical tool for tailoring evidence around upper extremity (UE) rehabilitation after a stroke. Methods: This study used a mixed methods (consensus approach), which includes a survey $(n=26)$ and nominal group meeting (NGM) with physiotherapists ( $n=15)$. Result: The first part of this study (survey) showed that 59 items (recommendations) from the UK National Clinical Guidelines for Stroke around UE post-stroke rehabilitation were accepted as consensus. Of these fifty-nine items, only 5 were rated as "no consensus." In addition, of all the items that had been included, not a single one was rejected. The results led to three groups of recommendations based on the agreed median scores. These are as follow: lack of agreement (no consensus), highest consensus with tightest range of scores, or lowest consensus with widest range of scores. The second part of this study (NGM) explores four themes, which appeared to significantly influence the concept of "tailoring" and the participants' ways of thinking. Conclusion: This study illustrates the role of tailoring in bridging the time-gap between the evidence and its implementation in the clinical setting. The study also provides more clarification about the role of tailoring and outlines the steps required to effectively investigate it.
\end{abstract}

\section{Author Bio(s)}

Salem F. Alatawi, MSc, PhD, is an assistant professor of neurorehabilitation, Physical Therapy Department, Faculty of Applied Medical Sciences, Vice-dean of the deanship of graduate studies at University of Tabuk - Saudi Arabia.

\section{Acknowledgements}

I would like to thank the University of Tabuk- Saudi Arabia for its generous support. I also would like to thank the physiotherapists at the neurorehabilitation units in Tabuk-Saudi Arabia, for their participation in this study and Mr. Ahmad Al-Hefdhi for his support. 


\title{
TIIAHSP \\ The Internet Joưnal of Allied Health Sciences and Practice
}

\author{
Dedicated to allied health professional practice and education
}

Vol. 17 No. 2 ISSN 1540-580X

\section{The Tailoring of National Guidelines for Upper Extremity Post-Stroke Rehabilitation: A Concept in Use}

\author{
Salem F. Alatawi \\ University of Tabuk \\ Saudia Arabia
}

\begin{abstract}
Background: In Saudi Arabia, national evidence-based clinical guidelines (NCG) were created based for healthcare interventions. However, clinicians appear to be slow to incorporate the evidence into practice. As a result, there exists a time-gap between the gathering and presentation of evidence for best practice and the use of that evidence by clinicians. It becomes, therefore, imperative to investigate the gap between the research evidence and clinical practice. The knowledge to action (KTA) framework is available to facilitate the implementation of research evidence. This framework incorporates the theory of tailoring, a concept that is intended to create a fit between the evidence for the intervention and its use by the clinician. Unfortunately, the process of tailoring stills awaits clear guidelines on how it should be implemented. Consequently, this study presents hitherto unknown strategies for using novel methods, which reflect the particular role of the KTA framework in tailoring recommendations. Aim: The purpose of this study is to examine the use of the UK National Clinical Guidelines for Stroke as a clinical tool for tailoring evidence around upper extremity (UE) rehabilitation after a stroke. Methods: This study used a mixed methods (consensus approach), which includes a survey $(n=26)$ and nominal group meeting (NGM) with physiotherapists $(n=15)$. Result: The first part of this study (survey) showed that 59 items (recommendations) from the UK National Clinical Guidelines for Stroke around UE post-stroke rehabilitation were accepted as consensus. Of these fifty-nine items, only 5 were rated as "no consensus." In addition, of all the items that had been included, not a single one was rejected. The results led to three groups of recommendations based on the agreed median scores. These are as follow: lack of agreement (no consensus), highest consensus with tightest range of scores, or lowest consensus with widest range of scores. The second part of this study (NGM) explores four themes, which appeared to significantly influence the concept of "tailoring" and the participants' ways of thinking. Conclusion: This study illustrates the role of tailoring in bridging the time-gap between the evidence and its implementation in the clinical setting. The study also provides more clarification about the role of tailoring and outlines the steps required to effectively investigate it.
\end{abstract}

Keywords: stroke, upper extremity, rehabilitation, national clinical guidelines, implementation, knowledge translation 


\section{BACKGROUND}

The quest to deliver care based on best evidence led to greater awareness of the importance of evidence-based practice (EBP). ${ }^{1}$ The purpose of evidence-based practice is to ensure healthcare is backed by evidence that is firmly grounded on proven research and which includes the needs and preferences of individual service users as well as the expertise clinicians. ${ }^{2}$ Researchers, academicians, and clinicians must work together to close the gap between evidence and practice. Closing the gap requires the identification of what, why, and how the evidence is or is not being used in real world settings and to test approaches that improve the implementation of evidence-based practice. $^{3}$

One of the most effective implementation strategies is known as the National Clinical Guidelines (NCG). It is considered by many as an important strategy to facilitate evidence-based practice in physiotherapy. ${ }^{4}$ Fundamental questions now must be asked as to why, despite the national guidelines setting out the evidence for the healthcare interventions, this evidence base continues to grow while the implementation within practice often remains slow in responding? The discrepancy indicates the presence of a gap between the creation of evidence for best practice and the use of evidence within day-to-day practice.

Effective implementation strategies have the potential to improve the quality of service delivery. In 2006, Grimshaw et al suggested that guideline dissemination and implementation strategies can lead to improvements in care. ${ }^{5}$ Harrison et al extended this view in 2010 by proposing that health care based on best available evidence occurs with the tailoring and implementation of practice guidelines. ${ }^{6}$ Straus et al, however, believe that the key to improving the uptake of evidence-based care interventions is to move beyond simple dissemination of knowledge to the use of knowledge, which in turn, leads to closing the gap between evidence and care delivery. ${ }^{7}$

Clinical guidelines are based on high quality clinical research with contributions from clinical experts and patients, including the act of balancing benefits and harms. Herbert et al suggested that a comprehensive, rigorous review of high-quality clinical research existed as a feature of clinical guidelines and systematic reviews. ${ }^{8}$ However, a number of differences among these studies were reported, such as the process of disease management, the method of development, the role of the patient, and the validity plus time consumption.

Although the dissemination of the NCG in clinical practice can lead to improvement in the quality of service delivery, these guidelines are mostly general. They neither give detailed information about the how of chaining practice nor acknowledge the complexity of the process of implementation. Therefore, the gap between recommendations of NCG and using evidence in clinical practice remains. Authors emphasised that implementation of evidence-based guidelines among allied health care professionals (including physiotherapy) is a complex process where factors at both contextual (social, organisational, economic, and political) and individual level play an active role. ${ }^{9-11}$ This opinion was supported by Rycroft-Malone et al, who indicated that effective implementation of evidence into practice, such as guidelines, encompasses more than just the use of a standardised care approach in the way that it was originally intended. 12 It involves the process by which the targeted group becomes aware of, receives, accepts, and utilises the disseminated evidence.

Many researchers explored the impact of using clinical guidelines by physiotherapists. ${ }^{13-15}$ Unfortunately, they found that the creation of clinical guideline recommendations did not necessarily lead to standardized care approaches. The researchers noted that many factors might affect the proper application of evidence-based sources as clinical guidelines into clinical decision-making and physiotherapy practice. Other researchers argued that although physiotherapy has followed the example of other healthcare fields and produced many guidelines, physiotherapists are still behind the medical profession in evaluating the effect of guidelines and the awareness of various strategies intended to increase the use of guidelines in their practice. ${ }^{16-18}$ According to Herbert et al, physiotherapy groups have put a lot of effort and resources into the development process, but very few have followed this up with any systematic implementation and evaluation process. ${ }^{8}$

Several frameworks that facilitate the implementation of evidence are now available. The knowledge to action framework (KTA) is one example of these frameworks. ${ }^{19}$ The KTA framework, which was developed by Graham and colleagues and is termed the KTA cycle, provides an approach that builds on the commonalities found in an assessment of planed-action-theories. ${ }^{20}$ This framework, which has added a knowledge creation process to planned-action theories, has labelled the combined processes (planned action theories with knowledge creation) the KTA cycle. The first part of this cycle is knowledge creation, or the production of knowledge. This consists of three phases: knowledge inquiry, knowledge synthesis, and knowledge tools and/or product creation. The second part is the action cycle, and it contains seven action phases needed to implement knowledge in health care setting (see Figure 1-1). 


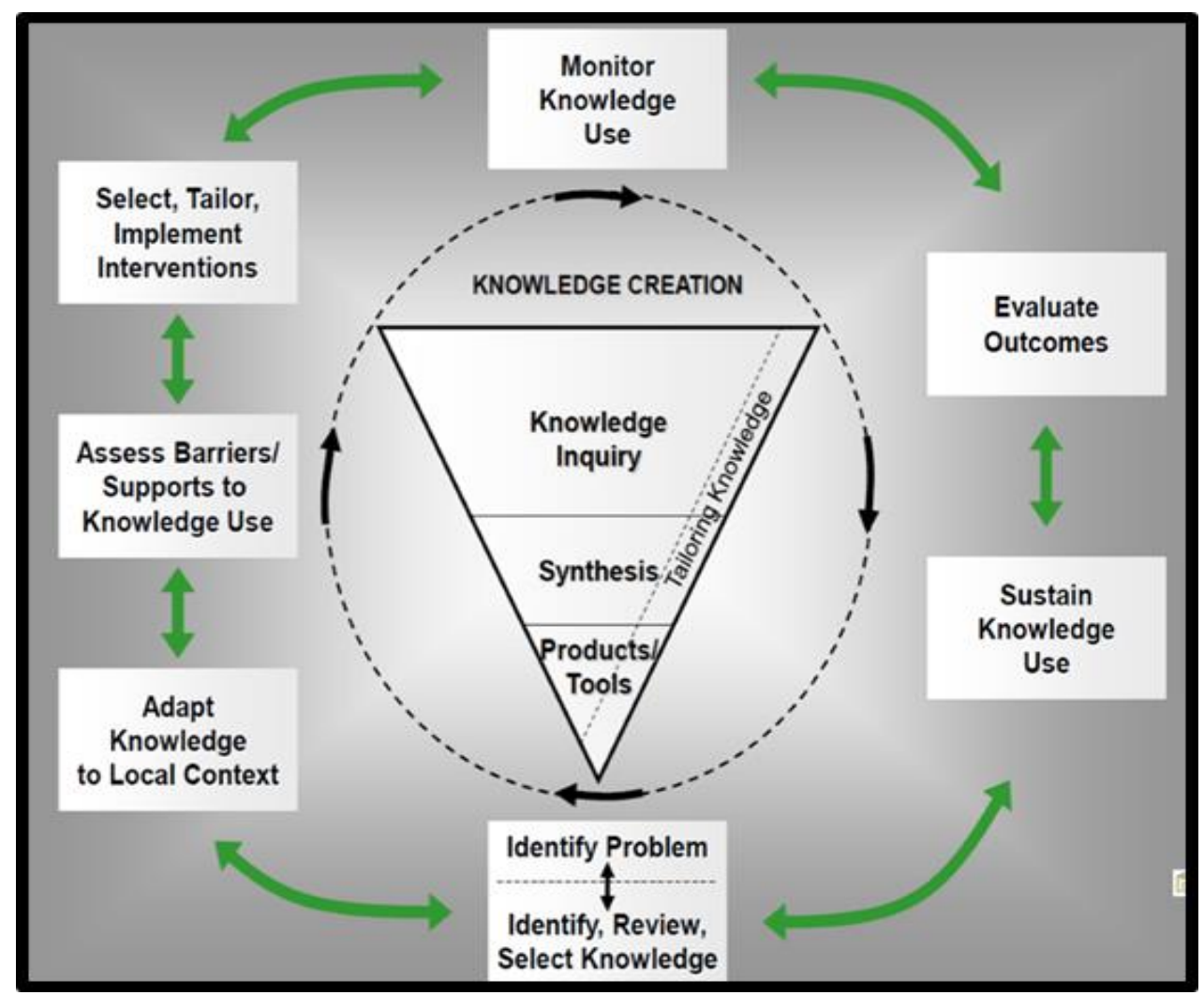

Figure 1-1: The knowledge to action (KTA) framework (Graham et al., 2006). The KTA framework comprises two distinct but related components: 1) knowledge creation (represented by the funnel) surrounded by 2) the action cycle. Each component involves several phases. Of particular interest in this framework is tailoring as an integral part of the knowledge creation.

Of particular interest to this concept analysis is the Graham et al KTA framework which incorporates tailoring as an integral part of the knowledge creation and implementation processes. ${ }^{20}$ Graham et al propose that knowledge or evidence can be tailored to the needs of potential users, and that evidence-based messages or recommendations can be tailored for a specific audience. However, the KTA process does not specifically prescribe what needs to be done at the tailoring phase.

A systematic study undertaken by Field et al reviewed studies between 2006 and 2013 that used the KTA framework in practice. ${ }^{21}$ The results showed that not a single one of the included studies used every phase of the KTA framework. Van Peppen et al sought to identify barriers to the use of guidelines in the Netherlands. ${ }^{22}$ They found that despite positive attitudes, physiotherapists infrequently used guideline recommendations in their day-to-day practice. This investigation highlights the downsides of merely publishing best practice guidelines and believing practitioners will adopt them into everyday practice. Similarly, in a systematic review, Van der Wees et al evaluated the effects of guideline implementation in physiotherapy. ${ }^{14}$ The authors recommended the need to set robust, specific, tailored implementation strategies based on the reported barriers and facilitators. Bernhardsson et al investigated the use of tailored guidelines implementation intervention in primary care physiotherapy in Sweden. ${ }^{23}$ The findings indicated that a tailored theory and evidence-informed, multi-component intervention had a positive effect on self-reported awareness of the existing guidelines. However, no effect for tailored multi-component intervention was recorded in relation to attitudes to implementation of guidelines. Similarly, Rutten et al investigated the application of the framework of intervention mapping of physiotherapy guidelines for the management of low back pain. ${ }^{24}$ The authors concluded that using the framework of intervention mapping provided a sound rational for the development and implementation of clinical practice guidelines. However, although the authors recognized that implementation of clinical guidelines might be affected by many factors at different levels, only environmental influences for patient behaviour and health outcome were employed in this framework.

In physiotherapy practice, one of the most common post-stroke problems is UE impairment which strongly effects the ability to perform activities of daily living (ADL) as well as quality of life. These symptoms are seen in $90 \%$ of patients affected by stroke. ${ }^{25}$ Upper extremity impairment can also adversely affect the rehabilitation and restoration of normal post-stroke functions. However, rehabilitation in this area is complicated by the multifactorial nature of UE 
problems which may operate across anatomical, functional, and social levels. Moreover, interventions are still controversial and inconclusive as variations exist between patients groups and outcome measures. Korner-Bitensky suggested that the aim of early stroke rehabilitation could be summarised as preventing post-stroke complications, minimising impairments, and maximising functions. ${ }^{26}$ However it is still a challenge to choose the most effective approach. Connell et al argued that the methods currently in use for UE rehabilitation are not enough towards maximizing post-stroke recovery and towards creating neural reorganizations. ${ }^{27}$ The authors concluded that future research can examine how stroke survivors in the rehabilitation setting can maximize work on UE therapy, as the power for neurological recovery is highest at this period. Another study by Garcia-Vega et al sought to develop a consensual approach amongst therapists (9 physiotherapists and 13 occupational therapists) regarding the usual care in the management of moderate to severe UE deficits post-stroke. ${ }^{28}$ The findings showed that a consensus on the usual care for moderate to severe UE deficits within participating stroke service was developed for use. However, this template was not intended to provide the best practice or most evidence-based approaches to post-stroke UE rehabilitation. Consequently, it did not necessarily equate with international recommendations for management of the hemiplegic patient. In a systematic review, Baatiema et al investigated the implementation of modern evidencebased strategies for acute stroke administration..$^{29}$ The results revealed many barriers and hurdles in the application of the latest methods, including the capacity for organizational change, institutional issues, lack of funds, lack of awareness of professionals, and lack of awareness in patients and relatives. They concluded that the causes for the slow application of the latest knowledge remain unknown and are yet to be understood.

In what concerns physiotherapy, the application of a guideline into practice would help investigate the process of tailoring. However, there is a considerable lack of information on how to tailor evidence within the discipline of implementation. In addition, the tailoring of best practice guidelines for UE can be affected by many factors. For example, individual factors such as, the knowledge the team operates in, the type of organisation they work in, as well as the patients they treat. Tailoring the evidence from NCG that addressed the approach of post-stroke UE rehabilitation remains thus far, unavailable. Consequently, this study seeks to establish how tailoring can be used within the discipline of implementation and how physiotherapists go about tailoring, or adapting national guidelines (such as, the UK National stroke Guidelines) to meet their own needs and clinical work.

\section{METHODS}

According to Rycroft-Malone, the "consensus approach" method was chosen in order to identify tailoring phenomena. ${ }^{1}$ The consensus approach in this study included 3 phases: panel phase, survey, and nominal group meeting (NGM).

\section{Panel Phase}

In order to establish face validity of the survey instrument, ten participants (including a researcher) reflected the full range of physiotherapists who work with stroke rehabilitation including 4 academics and 6 clinical physiotherapists assessed the questionnaire. One hundred items (recommendations) from the UK national stroke guideline had been chosen in relation to UE rehabilitation. The participants were asked to answer the question as to whether or not each item reflected the role of physiotherapists in UE rehabilitation after stroke. The experts were also asked to find items in NCG which might overlap with each other, were thought to be irrelevant, or were integrated with included items. All items were rated. Thirty three items were given a "high" rating with respect the area of UE rehabilitation; 17 items were given a moderate rating, and nine items were given a low rating. All these were included in the final version of the questionnaire. The final version of the questionnaire comprised 59 items/recommendations (for questions). See Figure 1-2.

\section{Survey}

The constructed questionnaire comprised two sections. The first section captured personal information about the respondent (e.g. qualifications) whilst the second section of the questionnaire contained questions related to rate their agreement that potential items from the UK National Clinical Guidelines for Stroke should be included in a practice protocol for the UE post-stroke rehabilitation. In the second section of this questionnaire, 59 items (recommendations) have been drawn from the UK National Clinical Guideline for stroke (phase 1) to inform guidelines for physiotherapy practice around the UE post-stroke rehabilitation. Participants were asked to consider individually each single item on its own merit and determine to what extend they think the item should be included in a protocol of UE post-stroke rehabilitation. Each item was rated 1-9 on a Likert scale, where 1 represented the lowest agreement and 9 represented the highest (see Figure 1-3). The participants available for this study, comprised physiotherapists employed in stroke rehabilitation departments in Tabuk, Saudi Arabia. A list of addresses and emails of physiotherapists, who routinely work with stroke patients was provided by the manager of the physiotherapy stroke rehabilitation service in Tabuk, Saudi Arabia. It was considered that, in order to sufficiently represent the wide geographical area, all physiotherapists working with stroke rehabilitation patients should be invited to take part in the study (as there were only $n=26$ ). In this study, stroke rehabilitation services are presented 
through three district general hospitals $(\mathrm{KKH}, \mathrm{KGH}$, and $\mathrm{KSH})$. All three hospitals provide intensive neurological rehabilitation for people with disabilities resulting from neurological conditions. It must be noted that the majority of patients admitted to these hospitals have suffered a stroke. The online survey was subsequently sent out to physiotherapists via email between September 2017 and December 2017.

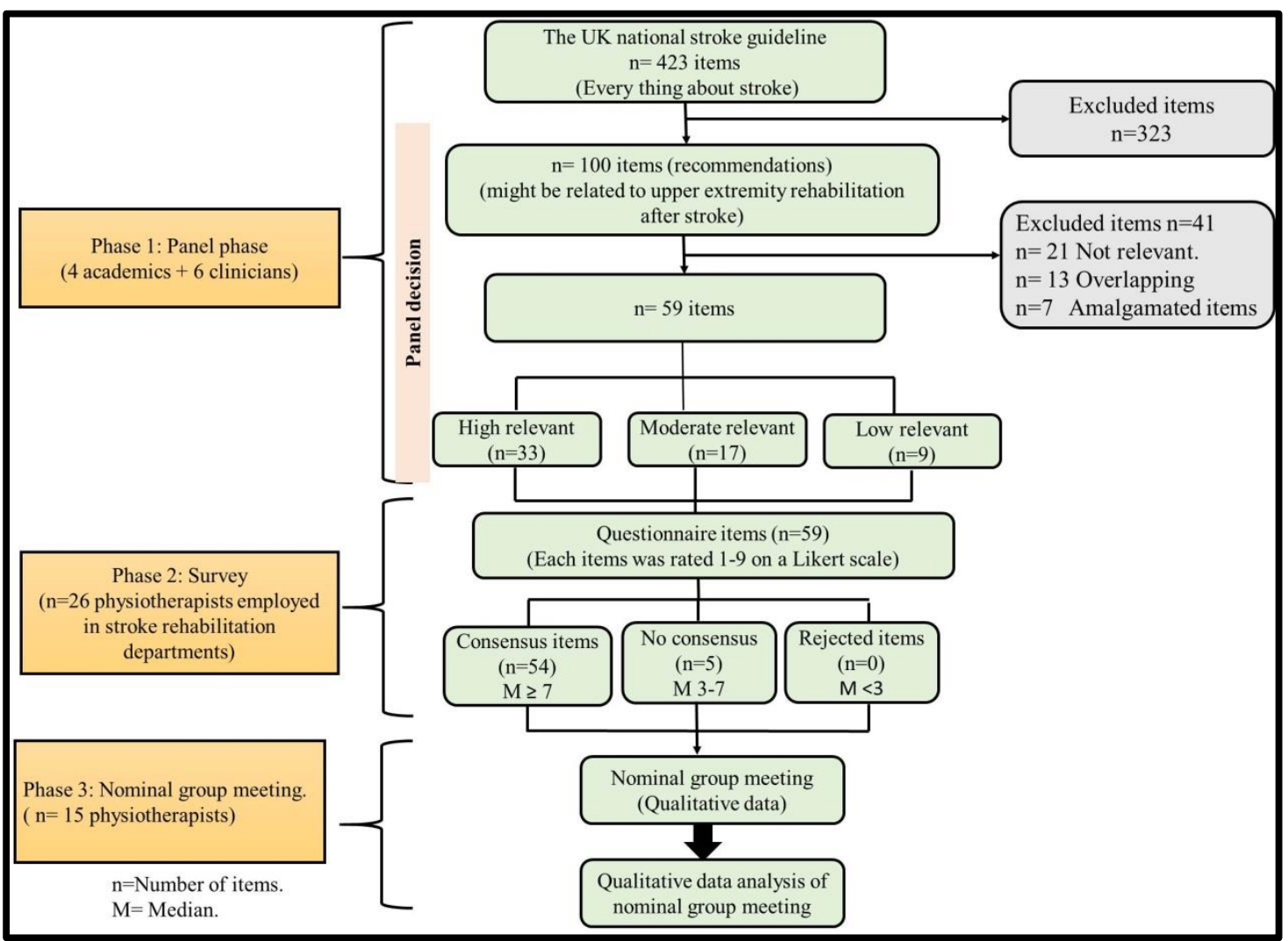

Figure 1-2: The process of consensus approach method

\section{Nominal Group Meeting}

The manager of physiotherapy stroke rehabilitation service was contacted and asked to identify staff from the three general hospitals who could attend a NGM. The final study sample for this part of the work consisted of 15 physiotherapists.

The NGM was arranged for when the completed questionnaires had been returned. The 59 items of NCG for stroke which the participants had already rated via the online survey (phase 2), were each discussed in the NGM. For each statement, the pattern of responses for the group was presented alongside each member's response to that statement. This allowed participants an opportunity during the NGM to see the spread of agreement and how their response related to that agreement. The recommendations already rated by the participants in the survey were discussed in turn. The focus of these discussions centered primarily on those items listed in the recommendations that were the source of the most disagreement (no consensus), and those items where agreement had reached the highest/lowest consensus. By generating "consensus" recommendations from NGT, the concept of "tailoring" was employed to explore the issues that shape how physiotherapists perceive the relevance of items.

\section{Data Analysis}

With regards to the survey, the frequency of responses to each item (standard) was calculated. Median and interquartile ranges were used and calculated for each statement by using SPSS. Standards with a median score of $<3$ were rejected on the basis that no consensus had been reached. Standards with a median score of $\geq 3$ and $<7$ were retained for the nominal group discussion, whereas standards with median scores of $\geq 7$ were both accepted and discussed in the NGM (Table 1-1). ${ }^{1}$ 


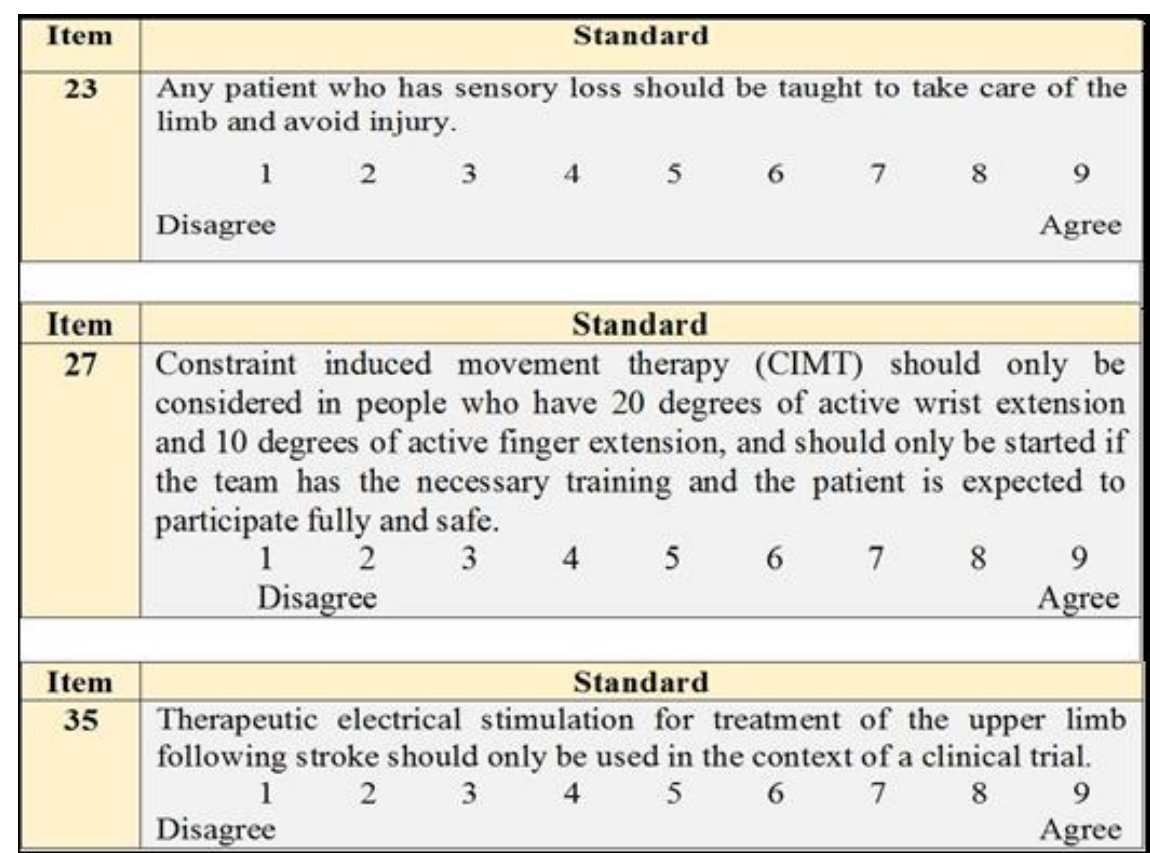

Figure 1-3: An example of part two of the questionnaire. This part of the questionnaire asks participant to think about how relevant potential items (extracted from the UK national stroke guideline) for an evidence based protocol for the UE rehabilitation might be. For each item, participant was asked to rate how strongly he agrees or disagree that each item should be included.

Table 1-1: Formal consensus process ${ }^{1}$

\begin{tabular}{|l|l|}
\hline Median value & Status \\
\hline$M \geq 7$ & Accepted 'consensus' \\
\hline$M$ 3-7 & No consensus 'go to nominal group meeting' \\
\hline$M<3$ & Rejected \\
\hline
\end{tabular}

The fact that NGM was the focus of the discussions held by participants of NGM provided those present with an opportunity to attest to the spread of agreement (findings from questionnaire), and the logic used by physiotherapists when scoring the items of NCG, as a way of understanding more about "tailoring." In addition, analysis of the qualitative data from the NGM discussion may assist in understanding the thinking behind the decision-making process which led to every recommendation. Data obtained from the audio recorded NGM were transcribed in full. The transcripts were analysed manually by conducting a qualitative content analysis in relation to the main research questions. The transcripts and notes were read and re-read to identify the themes. The themes on the transcript of the discussion of NGM were coded and categorized. Ethical approval was given.

\section{RESULTS}

Table 1-2 represents the socio-demographic characteristics of the respondents. These results indicate that the majority of respondents worked within the KSH (53.8\%). The majority of the survey respondents comprised $57 \%$ Bachelor of Science Degree (BSc) holders in physiotherapy, their highest academic qualification. In addition, $30.8 \%$ of the respondents had been worked as qualified physiotherapists for more than 15 years.

\section{Survey}

The response rate was very high and of the 26 questionnaires distributed, 26 were returned (100\%). Fifty- nine items from NCG for stroke around UE rehabilitation ( $n=59$ ) were accepted as "consensus," because they met the criteria (that is, a median score of 7-9), while only 5 items were rated as "no consensus." No included items were rejected (see Appendix).

The numbers in themselves were key to understanding the logic behind the physiotherapists' consensual score for each national clinical recommendation. The numbers also brought about an understanding of how physiotherapists go about adapting NCG with which throughout everything relevant to UE post- stroke rehabilitation. The results led to three groups of recommendations (Figure 1-4), based on the previously agreed median scores. These recommendations cover the following scores: lack of agreement (no consensus with the widest range of score (Figure1-4a), highest consensus with tightest range of scores (Figure1-4b) or lowest consensus with the widest range of scores (Figure1-4c). 


\begin{tabular}{|c|c|c|c|}
\hline Variable name & Characteristic & Number & Percentage \\
\hline \multirow[t]{3}{*}{ Area of practice } & KKH & 6 & $23.1 \%$ \\
\hline & KFH & 6 & $23.1 \%$ \\
\hline & KSH & 14 & $53.8 \%$ \\
\hline \multirow[t]{4}{*}{ Professional qualification } & Diploma & 6 & $23.1 \%$ \\
\hline & $\mathrm{Ba} / \mathrm{BSc}$ & 15 & $57.7 \%$ \\
\hline & MA/MSc & 5 & $19.2 \%$ \\
\hline & $\mathrm{PhD}$ & 0 & $0 \%$ \\
\hline \multirow{5}{*}{$\begin{array}{c}\text { Years of Physiotherapy } \\
\text { practice }\end{array}$} & $<1$ year & 5 & $19.2 \%$ \\
\hline & $1-5$ years & 5 & $19.2 \%$ \\
\hline & $5-10$ years & 5 & $19.2 \%$ \\
\hline & ears $15-10$ & 3 & $11.5 \%$ \\
\hline & $>15$ years & 8 & $30.8 \%$ \\
\hline
\end{tabular}

Table 1-2: Respondents variables professional characteristics $(\mathrm{N}=26)$.

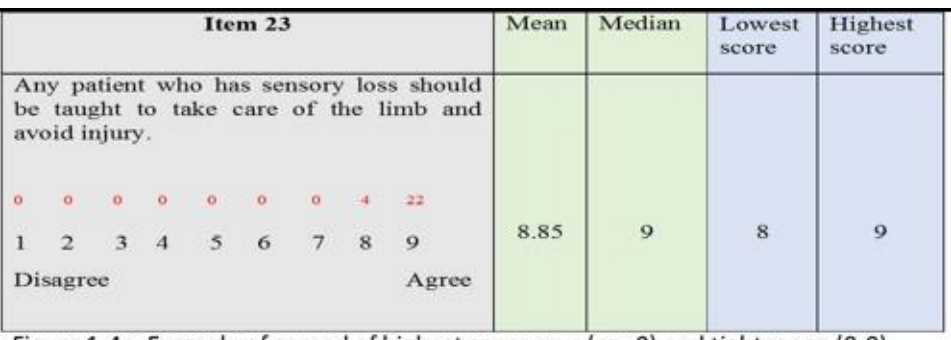

Figure 1-4a: Example of spread of highest consensus $(m=9)$ and tight range (8-9).

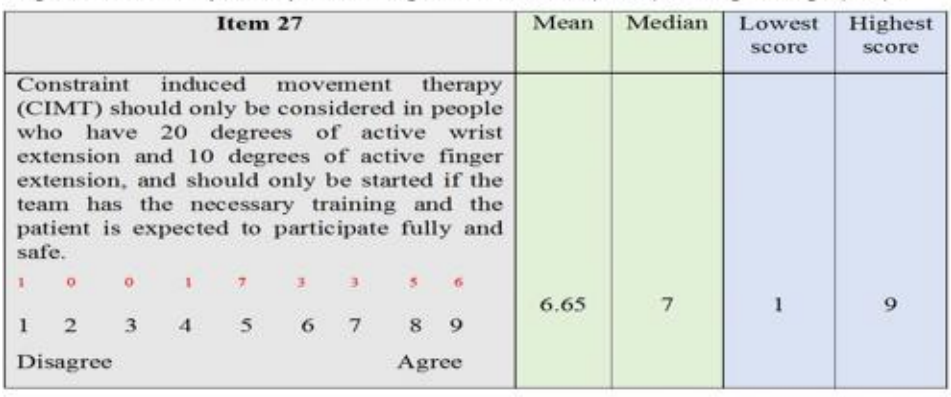

Figure 1-4b: Example of spread of lowest consensus ( $m=7)$ and wide range (1-9).

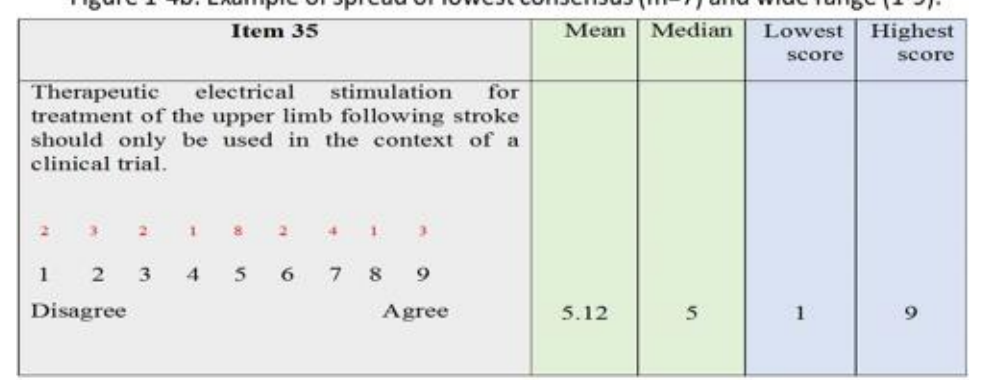

Figure1-4c: Example of no consensus ( $m=5$; go to nominal group meeting)

\section{Nominal Group Meeting}

The result explores four themes, which appeared to be significant influences on the concept of 'tailoring' and participants' ways of thinking at the time of rating NCG, to be relevant in their practice around UE post-stroke rehabilitation. These are as follows: context, implementer (end users), feasibility, and fidelity.

Context. A key factor emerging from the NGM was that availability of resources should be included in NCG around upper limb post-stroke rehabilitation in clinical practice. Participants were already thinking about the availability of technical resources at the level of organizations, which may help them shape implementation of guidelines in their practice. 
I'm aware they're out there but l've never been in a department where they were going to be on offer, so it's just like neutral.

Physiotherapists were very clear that "limited resources" was one impacting factor in transitioning some recommendations into practice. It was noticed that participants made frequent references to those operational hurdles inherent to the organizational structure in which they worked. Interrelated shortages of money, equipment, and resources access significantly impacted the way in which physiotherapists shaped their views of recommendations for UE post-stroke rehabilitation. For example:

If you haven't seen one and you have no access to it, you just don't think about it, even though you might go to a conference or read a paper and think about it

Participants also recognized that working with the patient's own resources influenced their way of thinking about decision-making around UE post-stroke rehabilitation. For example:

"... there may be evidence to support it but, so to speak, you can't necessarily access the equipment, and these patients agree to buy the stuff privately. Well, not everyone has the resources to do that."

Physiotherapists acknowledged that this dilemma "influence of context" went missing within the recommendations, which did not require equipment to be applied in clinical practice. Physiotherapists recorded a strong consensus for those items not needing equipment as compared to those items where equipment was required. For example, the following statement recorded a median of 9: "Healthcare professionals should be given training on how to position patients correctly after stroke."

"You don't need any equipment for this recommendation. You just need your hands."

Implementer (end users). Participants agreed that previous history or "previous exposure" to this intervention early in their career, but in different departments, might also act to shape their views around the implementation of recommendations in the clinical practice. This might explain the variation of scoring of consensus around particular recommendations between participants. Previous history could involve learning new skills, training, and the use of relevant interventions. For example, participants talked about how factors such as training and education impacted upon their practice in dealing with current dilemmas.

If you've never been trained in one thing, you've never used it, you don't know its potential of success with your patients.

The integration of the evidence and personal experience was presented in the tailoring of evidence around UE poststroke rehabilitation in daily practice. During the discussions, which were held at this NGM, the importance of clinical experience to gauge available evidence in clinical practice was noted. Participants found that the "clinical experience" was useful in helping with the decision-making process and in illustrating the relevant evidence in which they base their practice. However, there was a mismatch between evidence and personal experience. For example:

"I know the evidence quite well. I can see that it doesn't work for the function, but it does work for some form of strengthening, and some form of stabilization, things like subluxation".

The example above demonstrates the usefulness of clinical experience as a tool for decision-making, accentuated by the need to ascertain whether or not the recommendations can work at the level of clinical practice around UE post-stroke rehabilitation. Another example suggests that the physiotherapist's level of experience plays a unique and essential role in shaping the decision-making process by providing knowledge about tailoring the prioritization of therapy, such as treatment modality and its adjunct.

I think it's using it in context, and knowing what it does and doesn't do, but it can be relevant as an adjunct. So the words refer to the guidelines dealing with functional recovery. However, electrical stimulation is not indicated as a treatment modality, but rather as an adjunct to everything else. We're completely within the evidence base for using it, that's my interpretation, and that's why I use it.

Participants recognized that the effectiveness of clinical practice is hierarchical in nature and that randomized controlled trails (RCTs) are perceived as the 'gold standard'. However, they agreed that the hierarchies of evidence (such as RCTs) were of minimal relevance to physiotherapy. In addition, Participants identified that NCG traditionally 
relied on some type of research, and that usually randomized, controlled studies on interventions are used to make statements about "what works>" There was also an element of mismatch between decision-making with respect to, on the one hand, an RCT which focuses on overall effectiveness for groups of patients, and on the other, at individual level, their ways of working in daily clinical practice. For example:

The problem with rehabilitation research and evidence is that it's so pinned to RCTs, and just because in an RCT it doesn't work for five out of the eight patients, the three out the eight patients for whom it worked for, constitute the three that are excluded. They're the eight patients we're seeing, okay it didn't work for five, but on what logic aren't we going to offer it to the three for whom it was also meant to work.

In this NGM, it was clear that physiotherapists did not equate evidence-based practice with the research process. Some physiotherapists believed that other types of research evidence, such as qualitative research, could constitute an important source within evidence-based practice in the field of physiotherapy.

Even in physio, if you look at the journals over the past two years, the amount of more subjective, qualitative data is coming in by the bucket loads, it's just not being used in many of the guidelines. But it is starting to come in, so hopefully we are moving a bit more in that direction.

Feasibility. One of the central issues in the shaping of NCG around UE post-stroke rehabilitation was feasibility. Feasibility was perceived as a pattern when thinking about the intervention and whether it was "feasible" or not to be tailored for real practice. It was clear that physiotherapists shape their recommendations for UE poststroke rehabilitation not only on the visibility of evidence, but also on the regular use of this evidence in clinical practice.

If it's not common, if it's not on the shelf you tend to forget about it, it's not in your common artillery is it? It's just there filling the space, and then something might jog your memory, like coming in and thinking 'This could use a bit of tape', you know what I mean? It just reminds you of modalities that at the time, may be out of your sight of mind, so to speak.

Potential applicability and appropriateness of the choice of evidence to suit local circumstances in clinical practice were recognized as important factors in shaping implementation of evidence relative to UE post-stroke rehabilitation. The participants in the NGM emphasized the feasibility of applying the evidence in clinical practice, as there is some evidence for specific interventions that would be difficult to apply technically for a number of reasons.

The thing with subluxation, when you look at the evidence, you have to have it on for a high number of hours, and you have to keep putting it on, and actually when you take it off, it doesn't show a carry over. That's what the evidence shows currently, but if you can concurrently work to build up the strength, it can be an adjunct. So again, it's the way you use it; the evidence is there for some things and not for others.

Fidelity. Participants unanimously acknowledged that implementation of evidence around UE post-stroke rehabilitation is a complex process contingent upon numerous factors. One of these factors can be found at the level patient and constitutes just such a problem. This factor might influence the thinking process around the implementation of a particular recommendation. Physiotherapists observed that patients' complaints over certain aspects of care constituted one of the main elements which might shape the implementation of recommendations for UE rehabilitation. One participant said:

It's people with cognitive issues that might not benefit from, for instance, some settings within the rehab. They might be compliant with certain things, but if they haven't got the carers by their side, that are physically with them, they might not be appropriate for the Botox.

Physiotherapists also acknowledged that applying some interventions in their current working place would be difficult. However, these interventions could be applied at different clinical locations, at different times, and with different patients. In the meantime, although this intervention might work positively for patients, fidelity issues with regards to how the intervention was implemented might be raised. What might also come to the fore is the acceptability and/or availability of using this intervention in the current area of practice. To ensure fidelity, intervention activities are required to be implemented as intended, usually with similar participants, in an identical type of setting as that in the research trials. In addition, it has been stated that the patient's voice is conclusive in accepting a potential intervention. This, in turn, might play an important role in empowering individual therapists with the means to integrate research evidence into their practice. For example, participants admitted that the voice of the patient was important to implement national clinical recommendations. 


\section{It does work for some people in a huge way.}

Clinical guidelines highlight evidence of the effectiveness of applying UE post-stroke rehabilitation. While this might have a positive effect in increasing awareness of a range of knowledge information in the clinical practice, the application of this evidence in clinical practice might be as ambivalent as in trying to understand the mechanism of action during practice. For example, physiotherapists emphasize that some items in clinical guidelines cause an element of consternation amongst practitioners during clinical practice for UE post-stroke rehabilitation. It was clear that in some cases it was difficult for the physiotherapists to understand the mechanism under which the intended intervention had been effectuated.

You see I wonder about that, you see one can reach a conjecture; is that acupuncture, for pain in a post-stroke shoulder, or is it at acupuncture for recovery because there are two different purposes for its' use.

\section{DISCUSSION}

Findings from this study illustrated that the notion of "tailoring" of recommendations for UE post-stroke rehabilitation was linked simultaneously to several factors. This includes the context, the engagement of end users, the nature of practice, the complexity of evidence, the feasibility, and fidelity.

\section{Context}

Tailoring is a concept designed to create a fit between the intervention and the context into which the intervention is being implemented. The findings indicated that "context," namely the environment or setting in which the proposed change is to be implemented, is a dominant factor in the tailoring of national stroke recommendations for UE poststroke rehabilitation. These findings are in agreement with others who confirmed that organizational factors played an important role in the decision-making process of physiotherapists.23,29 In addition, the findings showed that operational factors related to the running of an organization might act to restrict or facilitate the physiotherapists' engagement in the implementation of evidence into practice. Moreover, interviews held with physiotherapists emphasized the important role of organizations in providing education, training, and resources, and in allocating time for physiotherapists to integrate evidence into practice. Findings also highlighted a consensus over the dynamics and factors that shape the working environment. For instance, the availability of resources and organizational structure (embedded interventions) can determine and shape physiotherapy around UE post-stroke rehabilitation. When evidence is integrated into clinical practice, organizational characteristics can be viewed from various angles such as the structure, the culture, the human resources, or the policies of the organization.

\section{The Nature of Practice}

The precise process for effective tailoring of evidence for facilitating the role of physiotherapy in UE post-stroke rehabilitation has not yet been identified. Consequently, a blended method study including survey consensus work and NGM was used.

Some evidence was fairly straightforward in its incorporation into clinical practice, whereas other evidence proved complicated to the degree that it could not be addressed simply through the evidence base. To this effect, an example of the complications encountered at the level of evidence pertains to individual patient expectations and to the wider organizational and environmental influencing factors. Harrison et al maintain that tailoring implementation is gained through the use of multiple considerations such as, local needs, priorities, legislation, policies, and resources, which all influence the scope of practice within the local health service context. ${ }^{6}$ Graham et al emphasize that involving relevant decision makers, including clinicians, managers, researchers, and policy makers can provide an approach to adapting a guideline to a local context. ${ }^{10}$ The findings from this paper showed that physiotherapy work around UE post-stroke rehabilitation remains complex. For example, there was no consensus on the visualization and priority of treatment toward the UE post-stroke rehabilitation in real practice between practitioners. These findings reflect the nature of the complexity that occurs when trying to implement evidence about UE poststroke rehabilitation in clinical practice. The complexity reflects the level of engagement of the findings and the needs of the particular knowledge user in the implementation of EBP.

\section{Feasibility}

Feasibility was another important consequence of the tailoring process which, perhaps, raises the need to consider the local adaptation of NCG into local practice. Tailoring can be used to design implementation strategies to overcome identified barriers in order to create a better fit to the local setting. This way, it will help promote greater acceptance, use, and sustainability of the evidence implementation process within the targeted group or context. Guideline adaptation therefore, needs to fit within existing models of delivery in the targeted setting. Harrison et al 
indicated that a lack of fit with existing models of delivery could create end users ambivalence and barriers to guideline adaptation in everyday practice. ${ }^{6}$

A study by Van Peppen et al found that physiotherapists infrequently used guideline recommendations in their daily practices. ${ }^{22}$ This led to recommendations for setting specific, tailored implementation strategies based on reported barriers and facilitators to create an effective modus operandi for day-to-day practices. Findings from this study showed that a particular obstacle to research was one of the common barriers in the implementation of evidence into practice. For example, while the findings identified that RCTs are reported as the gold standard of evidence in most physiotherapy literature, many clinical questions remained unanswered in real practice. The findings from the consensus work highlighted that hierarchies of evidence (such as RCTs) were of minimal relevance to physiotherapy practice. They also showed that NCG traditionally relied on some type of research, usually RCTs. Some physiotherapists perceived other types of evidence, such as qualitative evidence, as being potentially of high influence in the implementation of evidence into practice.

\section{Fidelity}

Understanding how guidelines can effectively target the local context of care is a first step in implementing evidence into practice. Furthermore, customizing a clinical practice guideline for a particular organization may help improve acceptance and adherence. However, customizing a guideline to local conditions might create a fundamental challenge to the point where the tailoring of guidelines to local context will depart from its evidence base. This challenge raises serious questions about the quality and validity of the recommendations. It must be noted that no validated process for guidelines adaptation has been documented. However, specific needs, priorities, legislation, policies, and resources in the targeted setting should be considered in clinical guidelines adaptation to local clinical practice. To ensure fidelity, intervention activities must be implemented as intended, usually with a similar population in the same type of setting as in a research trial. In the tailoring process, it was important to engage end users in adapting evidence-based interventions for their particular setting and consequential adoption of interventions. 2,20 However, there was a concern that in the tailoring process, involving end users of an adapted guideline risks diluting the original message and with it the fidelity of the evidence. Graham et al propose that the key to successful implementation is to ensure that there is a match between the expected research findings, the targeted knowledgeusers, and the selected implementation intervention strategies. ${ }^{20}$

\section{Limitations}

A potential limitation or weakness of the current research was that there were only 15 physiotherapists working routinely with stroke rehabilitation who contributed to the study, which may limit generalizability. A wider sample in the future would expand our understanding of the implementation process in physiotherapy practice. Furthermore, the second round meeting with NGM was another limitation in this study. A future study may need to focus on the 'timing of tailoring' such as, the start and end of time of tailoring throughout the implementation process.

\section{CONCLUSION}

In conclusion, a key strength of this study centres around the exploration of the concept of "tailoring" through the KTA framework, as well as through a consideration of "tailoring" in relation to the implementation context. Tailoring evidence-based guidelines to a particular context facilitated a robust process, which guaranteed that the end product met the needs of the particular context. The focus is on a local context and practice when adapting and implementing evidence-informed interventions (practices, programs, policies). The nominal group technique proved effective in offering a formal consensus approach for including relevant NCG about UE post-stroke rehabilitation where rigour and transparency constituted imperatives and not options. ${ }^{1}$ One of the key features of NGM technique is to examine a phenomenon from many points of view, looking for new ideas and insights that will not only explain what is happening but also what is hindering the acceptance of the new technique. The KTA framework emphasises the interactions between researchers and knowledge users. Thus, embedding in this study a consensus approach through NGM provides a good window for understanding the concept of 'tailoring' in the KTA framework. It provided an opportunity for collaborative working between physiotherapists and researchers throughout all stages of the guideline development process. This collaborative approach may enhance the credibility of the guideline in the eyes of the end users. As a result, physiotherapists can be empowered to participate in research and thus have a voice in informing policy and practice. The method outlined proved to be a practical and systematic way of integrating a number of different evidence sources. The resultant guideline is a mixture of research-based and consensus-based recommendations. This method outlined how to blend research involving expert opinion and end users.

In summary, there were 3 primary findings from this study. First, tailoring is an active, interactive process that takes place in practice. Second, tailored implementation strategies constitute those designed to overcome identified barriers and to enhance enablers within the specific setting. Third, a key concern with tailoring evidence-based guidelines is how to maintain a balance between feasibility and fidelity. 


\section{References}

1. Rycroft-Malone J. Formal consensus: the development of a national clinical guideline. Qual Health Care. 2001;10(4):238-244.

2. Graham I, Harrison M, Brouwers M, Davies B, Dunn S. Facilitating the use of evidence in practice: Evaluating and adapting clinical practice guidelines for local use by health care organizations. J Obstet Gynecol Neonatal Nurs. 2002;31(5):599-611.

3. Peter D, Adam T, Alonge O, Agyepong I, Tran N. Implementation research: What it is and how to do it. BMJ. 2013;347:f6753.

4. Nilsagard $Y$, Lohse G. Evidence-based physiotherapy: a survey of knowledge, behaviour, attitudes and prerequisites. Adv Physiother. 2010;12(4):179-186.

5. Grimshaw J, Eccles M, Thomas R, MacLennan G, Ramsay C, Fraser C, Vale L. Toward evidence-based quality improvement. Evidence and its limitation of the effectiveness of guidelines dissemination and implementation strategies. J Gen Intern Med. 2006;21(2):14-20.

6. Harrison M, Legare F, Graham I, Fervers B. Adapting clinical practice guidelines to local context and assessing barriers to their use. CMAJ. 2010;182(2):78-84.

7. Straus S, Tertroe J, Graham I. Knowledge Translation in Health Care. Moving from Evidence to Practice. 2nd ed. Oxford: Wiley-Blackwell BMJ; 2013.

8. Herbert R, Jamtvedt G, Hagen K, Mead J. Practical Evidence-Based Physiotherapy. 2nd ed. Edinburgh: Elsevier Butterworth Heinemann; 2011.

9. Bridges $P$, Bierema L, Valentine $T$. The propensity to adopt evidence-based practice among physical therapists. BMC Health Serv Res. 2007;7(1);103-112.

10. Ploeg J, Davies B, Edwards N, Gifford W, Miller P. Factors influencing best-practice guideline implementation: Lessons learned from administrators, nursing staff, and project leaders. Worldviews Evid Based Nurs. 2007;4(4):210-219.

11. Grol R, Wensing M. What drives change? Barriers to and incentives for achieving evidence-based practice. Med J Aust. 2004;180(6):57-60.

12. Rycroft-Malone J, Fotenia M, Bick D, Seers K. Protocol-based care: Impact on roles and service delivery. J Eval Clin Pract. 2008;14(5):867-873.

13. Bekkering G, Engers A, Wensing M, Hendriks H, van Tulder M, Oostendorp R, Bouter L. Development of an implementation strategy for physiotherapy guidelines on low back pain. Aust J Physiother. 2003;49(3):208214.

14. Van der Wees P, Moore A, Powers C, Stewart A, Nijhuis-van der Sanden M, De Bie R. Development of clinical guidelines in physical therapy: Perspective for international collaboration. Phys Ther. 2011;91(10):1551-1563.

15. Hanchard N, Goodchild L, Thompson J, O'Brien T, Davison D, Richardson C. Evidence-based clinical guidelines for the diagnosis, assessment and physiotherapy management of contracted (frozen) shoulder: Quick reference summary. Physiotherapy. 2012;98(2):117-120.

16. Van der Wees $P$, Jamtvedt G, Rebbeck T, de Bie R, Dekker J, Hendriks E. Multifaceted strategies may increase implementation of physiotherapy clinical guidelines: a systematic review. Aust J Physiother. 2008;54(4):233-241.

17. Menon A, Korner-Bitenskey N, Kastner M, McKibbon K, Straus S. Strategies for rehabilitation professionals to move evidence-based knowledge into practice: a systematic review. J Rehabil Med. 2009;41(13):1024-1032.

18. Dannapfel $P$, Peolsson A, Nilsen P. What supports physiotherapists' use of research in clinical practice? A qualitative study in Sweden. Implement Sci. 2013;8(1):31-52.

19. Rycroft-Malone J. Using theory and frameworks to facilitate the implementation of evidence into practice. Worldviews Evid Based Nurs. 2010;7(2):57-58.

20. Graham I, Logan J, Harrison M, Straus S, Tetroe J, Caswell W, Robinson N. Lost in knowledge translation: Time for a map? J Contin Educ Health Prof. 2006;26(1):13-24.

21. Field B, Booth A, llott I, Gerrish K. Using the knowledge to action framework in practice: a citation analysis and systematic review. Implement Sci. 2014;9(1):172.

22. Van Peppen R, Maissan F, Van Genderen F, Van Dolder R, Van Meeteren N. Outcome measures in physiotherapy management of patients with stroke: a survey into self-reported use, and barriers to and facilitators for use. Physiother Res Int. 2008;13(4):255-270.

23. Bernhardsson $S$, Larsson $M$, Eggertsen $R$, Olsén MF, Johansson $K$, Nilsen $P$, Nordeman $L$, van Tulder $M$, Öberg, B. Evaluation of a tailored, multi-component intervention for implementation of evidence-based clinical practice guidelines in primary care physical therapy: a non-randomized controlled trial. BMC Health Serv Res. 2014;14(1):105. doi: 10.1186/1472-6963-14-105. 
24. Rutten G, Harting J, Bartholomew L, Braspenning J, van Dolder R, Heijmans M, Oostendorp R. Development of a theory-and evidence-based intervention to enhance implementation of physical therapy guidelines for the management of low back pain. Arch Public Health. 2014;72(1):1-12.

25. Smith M. Management of hemiplegic shoulder following stroke. Nurs Stand. 2012;26(44):35-44.

26. Korner-Bitensky N. When does stroke rehabilitation end? Int J Stroke. 2013;8(1):8-10.

27. Connell L, McMahon N, Tyson S, Watkins C, Eng J. Case series of a knowledge translation intervention to increase upper limb exercise in stroke rehabilitation. Phys Ther. 2016;96(12):1930-1937.

28. Garcia-Vega J, Gregory G, Lind C, Singer B. Development of a consensus approach to upper limb rehabilitation early post stroke amongst a cohort of Western Australian therapists. New Zealand Journal of Physiotherapy. 2016;44(3):133-147.

29. Baatiema L, Otim M, Mnatzaganian G, de-Graft Aikins A, Coombes J, Somerset S. Health professionals' views on the barriers and enablers to evidence-based practice for acute stroke care: a systematic review. Implement Sci. 2017;12(1). 
Appendix: The Mean and Median of Items of the Questionnaire.

Fifty-nine (59) items have been extracted to inform guidelines for physiotherapy practice for upper limb rehabilitation. Each statement was rated 1-9 on a Likert Scale where 1 represented least agreement and 9 represented most agreement. The frequency of response to each standard was calculated as well as the spread of agreement. Median and interquartile range was calculated for each statement. Standards with a median score of $<3$ was rejected on the bases that no consensus was reached. Standards with a median score of $\geq 3$ and $<7$ were sent to the nominal group meeting, whereas standards with median scores of $\geq 7$ were accepted.

\begin{tabular}{|c|c|c|c|c|c|c|c|c|}
\hline Items & Mean & Median & Items & Mean & Median & Items & Mean & Median \\
\hline 1 & 7.77 & 9 & 21 & 7.58 & 8 & 41 & 8.73 & 9 \\
\hline 2 & 8.85 & 9 & 22 & 7.88 & 8 & 42 & 8.65 & 9 \\
\hline 3 & 8.58 & 9 & 23 & 8.85 & 9 & 43 & 8.58 & 9 \\
\hline 4 & 8.08 & 9 & 24 & 8.04 & 8 & 44 & 5.81 & 6 \\
\hline 5 & 6.96 & 7 & 25 & 7.88 & 8 & 45 & 7.00 & 7 \\
\hline 6 & 8.54 & 9 & 26 & 8.73 & 9 & 46 & 8.77 & 9 \\
\hline 7 & 8.27 & 9 & 27 & 6.65 & 7 & 47 & 8.08 & 9 \\
\hline 8 & 8.08 & 9 & 28 & 7.88 & 8.5 & 48 & 7.96 & 8 \\
\hline 9 & 8.19 & 9 & 29 & 8.42 & 9 & 49 & 7.73 & 9 \\
\hline 10 & 8.73 & 9 & 30 & 8.50 & 9 & 50 & 8.54 & 9 \\
\hline 11 & 8.50 & 9 & 31 & 6.88 & 7 & 51 & 8.62 & 9 \\
\hline 12 & 6.69 & 7 & 32 & 8.54 & 9 & 52 & 8.23 & 9 \\
\hline 13 & 8.58 & 9 & 33 & 7.42 & 8 & 53 & 8.04 & 9 \\
\hline 14 & 8.85 & 9 & 34 & 5.23 & 5 & 54 & 8.08 & 8 \\
\hline 15 & 8.27 & 9 & 35 & 5.12 & 5 & 55 & 8.54 & 9 \\
\hline 16 & 8.38 & 9 & 36 & 4.62 & 5 & 56 & 8.65 & 9 \\
\hline 17 & 7.50 & 8 & 37 & 5.58 & 5 & 57 & 8.96 & 9 \\
\hline 18 & 8.00 & 8 & 38 & 8.12 & 9 & 58 & 8.85 & 9 \\
\hline 19 & 8.27 & 9 & 39 & 8.08 & 9 & 59 & 8.62 & 9 \\
\hline 20 & 8.08 & 9 & 40 & 8.19 & 9 & & & \\
\hline
\end{tabular}

